\title{
Ventricular Fibrillation During Amiodarone Infusion in a Patient With Wolff-Parkinson-White Syndrome and Atrial Fibrillation: A Case Report
}

\author{
Tiago Luiz Luz Leiria ${ }^{\mathrm{a}, \mathrm{c}}$, Augusto Mantovani ${ }^{\mathrm{b}}$, Rafael de March Ronsoni ${ }^{\mathrm{a}}$, \\ Leonardo Martins Pires ${ }^{\text {a }}$, Marcelo Lapa Kruse ${ }^{\text {a }}$, Gustavo Glotz de Lima ${ }^{a}$
}

\begin{abstract}
One third of Wolff-Parkinson-White Syndrome (WPW) patients develop atrial fibrillation (AF). Amiodarone is a commonly used drug for AF control in this scenario. The use of amiodarone can lead to Ventricular Fibrillation (VF) in some of these patients. Here we report and discuss a case of a WPW patient who went into cardiac arrest shortly after amiodarone infusion was started.
\end{abstract}

Keywords: Ventricular fibrillation; Atrial fibrillation; Wolff-Parkinson-White syndrome; Amiodarone; Procainamide

\section{Introduction}

Atrial fibrillation (AF) is frequent in patients with Wolff-Parkinson-White Syndrome (WPW). Accessory pathways with a very short anterograde refractory period may lead to rapid atrioventricular conduction resulting in AF degenerating into ventricular fibrillation (VF) [1]. Atrioventricular nodal blocking agents, such as verapamil, are contraindicated in rapid pre-excited AF because of their potential to accelerate anterograde conduction over the accessory pathway leading to VF. In this case report, we present a patient who came to the Emergency Department (ED) with pre-excited AF who developed VF during amiodarone infusion.

\footnotetext{
Manuscript accepted for publication May 10, 2012

${ }^{a}$ Instituto de Cardiologia/Fundacao Universitaria de Cardiologia Electrophysiology Service, Brazil

${ }^{\mathrm{b}}$ Universidade Federal de Ciencias da Saude de Porto Alegre, Brazil

${ }^{\mathrm{c}}$ Corresponding author: Tiago Luiz Luz Leiria, Servico de

Eletrofisiologia, Instituto de Cardiologia-RS, Av Princesa Isabel 395 ,

Office 203, Porto Alegre, Brazil. Email: tiagoleiria@yahoo.com
}

\section{Case Report}

A 31 year-old male patient arrived at the ED complaining of palpitations for the last hour. Clinical evaluation demonstrated no signs of hemodynamic compromise (blood pressure 130/80 mmHg; heart rate of $157 \mathrm{bpm})$. The electrocardiogram (ECG) revealed an irregular wide complex tachycardia with variable QRS duration in each beat and a forward axis on the horizontal plane. All this features were compatible with AF with ventricular pre-excitation (Fig. 1A). Due to the hemodynamic stability, intravenous amiodarone was administered (150 mg over first 10 minutes, followed by a dose of $1 \mathrm{mg} / \mathrm{min}$ ). The patient developed VF (Fig. 1B) soon after the bolus was terminated, which was reverted with electrical defibrillation. A sequential ECG showed WPW pattern with a left lateral pathway. The patient was referred for an electrophysiological study. Due to the initial presentation, radiofrequency ablation was indicated and it successfully terminated the conduction through the by-pass tract (Fig. 2).

\section{Discussion}

WPW consists of an accessory pathway between the atrium and the ventricle. There is a higher incidence of arrhythmias and sudden death in these patients. Sudden death incidence is $0.02 \%$ [2] per year. The mechanism of death is probably AF that degenerates into VF. AF affects approximately one third of patients with WPW [1]. The evolution to VF is rare; however, this may be precipitated by the use of nodal blocking agents, such as calcium channel blockers.

Amiodarone is a class III antiarrhythmic that is recommended for the management of pre-excited AF by the latest guidelines [3]. This makes amiodarone a commonly used drug for AF control in this scenario. However, Tijunelis and Herbert [4] published an article objecting to amiodarone indication as a first line treatment in WPW patients in AF. Simonian et al [5] also challenged amiodarone superiority over procainamide in controlling AF in WPW patients. They point out the fact that there is no properly conducted trial of amiodarone and procainamide that justify their use. Also, 

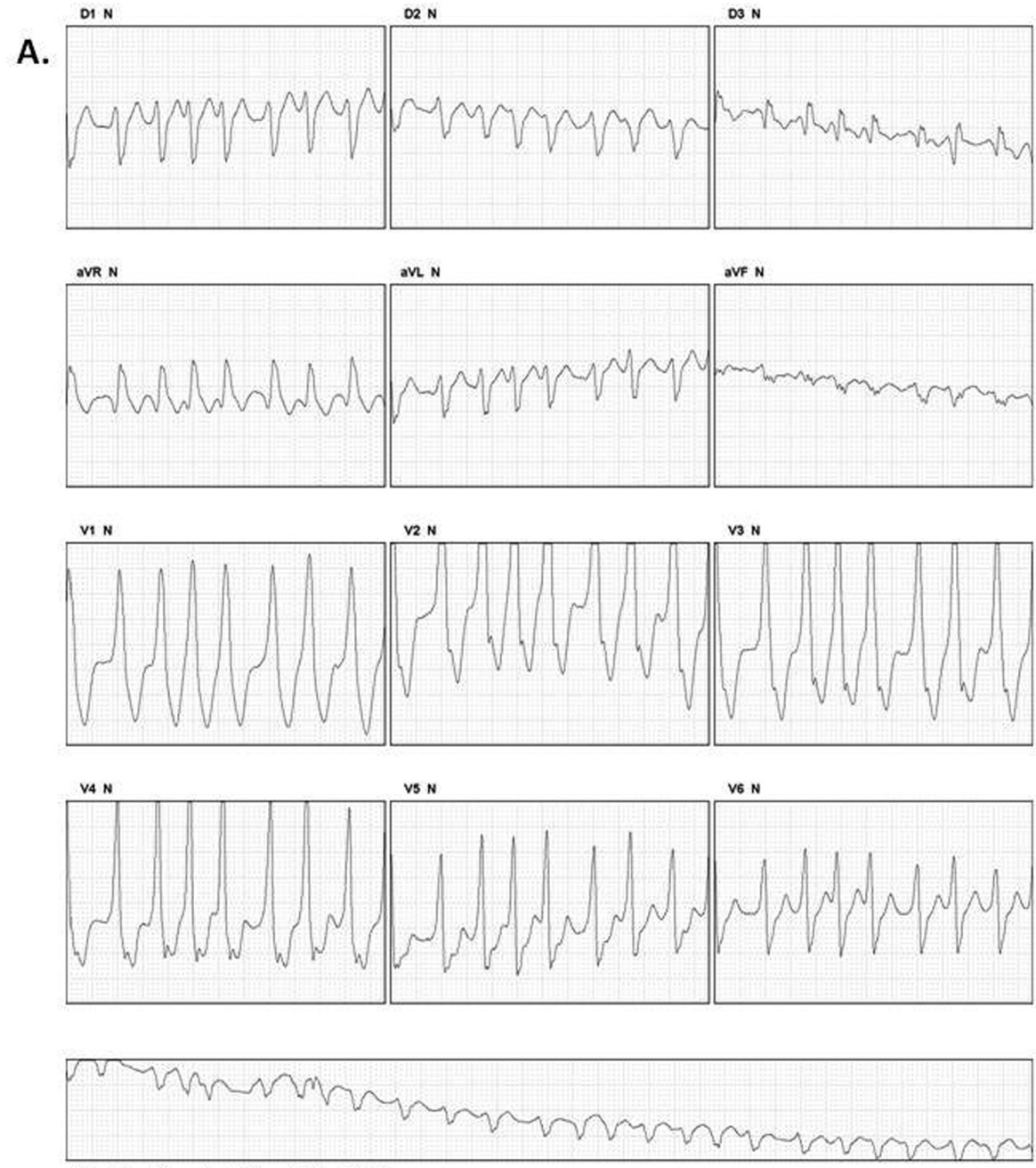

D2 N/2 $25 \mathrm{~mm} / \mathrm{s}$ Filtros: Rede MUSC

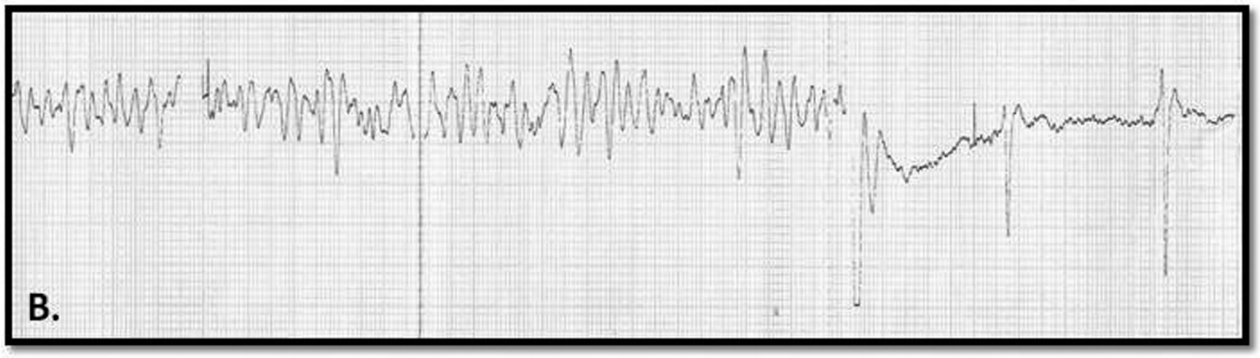

Figure 1. A) 12-lead-ECG showing pre-excited AF with fast conduction through the accessory pathway. The QRS morphology during AF suggests the presence of a left-lateral by-pass tract. B) VF Shortly after the amiodarone infusion started.

there are important differences between the drug dosages, routes of administration and primary outcomes in the currently available data [5].

In our case, amiodarone was promptly used in intravenous infusion to convert to sinus rhythm, to control the ventricular rate and prevent degeneration to VF. However, the case outcome was unexpected. There is evidence that until conversion to desethylamiodarone, intravenous amiodarone acts predominantly as a beta-receptor antagonist and, as such, blocking the AV node. This may explain why amiodarone is associated with conversion of AF to VF. However, the arrhythmogenic mechanism is not completely understood and the following hypothesis may explain this conversion: a) amiodarone may favor conduction over the accessory pathways by slowing or blocking conduction into the atrioventricular node and decreasing concealed retrograde conduction into the accessory bypass tract by normally conducted beats [6], b) hypotensive action of amiodarone or its solvent 


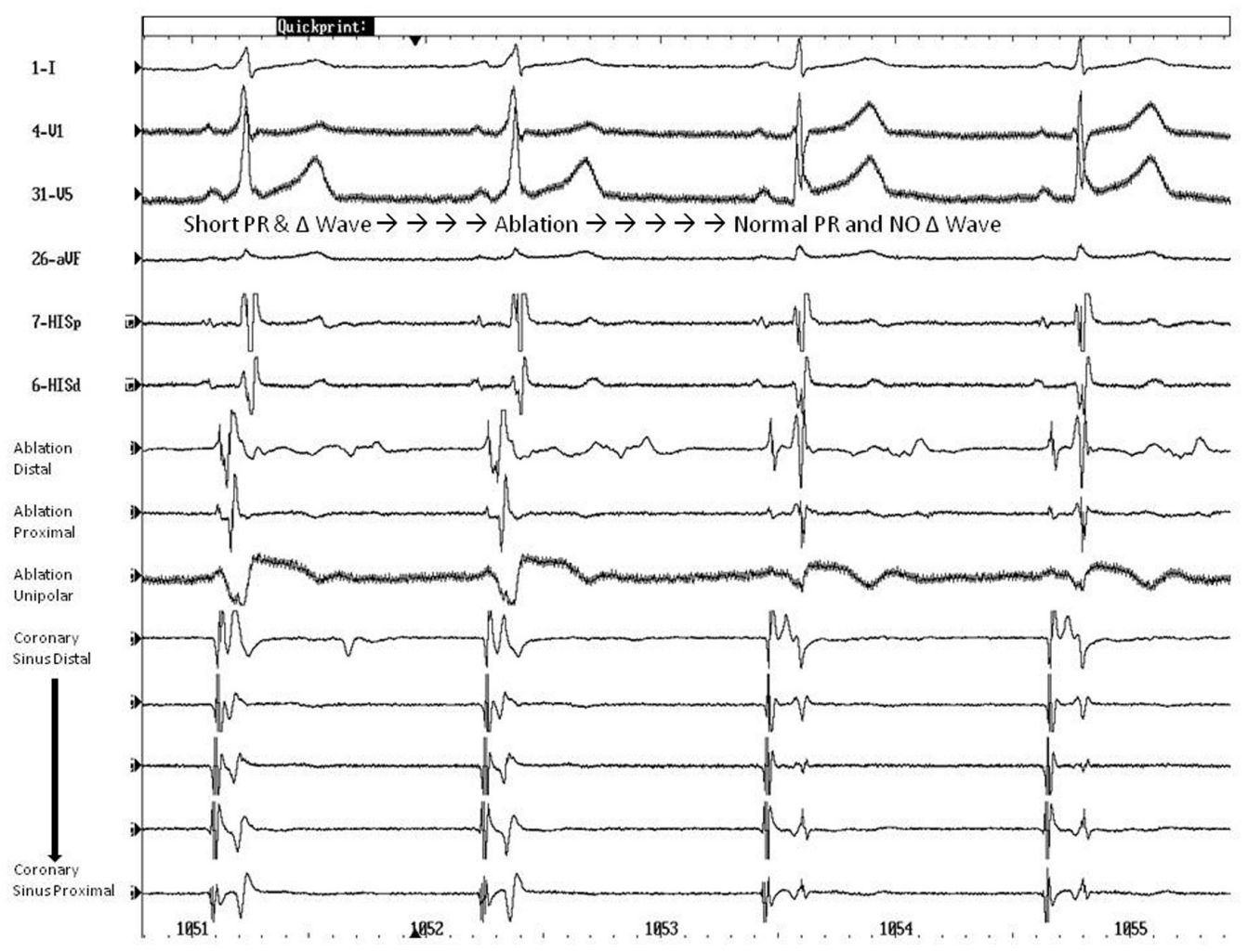

Figure 2. Electrophysiological study, confirming the presence of an accessory left lateral pathway. Radiofrequency ablation successfully terminated the conduction through the bypass tract.

which might trigger beta-adrenergic receptors, increasing the cardiac chronotropy and decreasing the anterograde refractory period of the accessory pathway [7].

The success rate of amiodarone infusion for converting $\mathrm{AF}$ to sinus rhythm is the same as placebo in the firsts two hours after the beginning of AF, although significant efficacy was found after 6 to 8 hours. Due to this fact, a meta-analysis compared amiodarone against flecainide at 1 to 2 hours, demonstrating that the latter drug is significantly more effective than amiodarone. Although at $24 \mathrm{~h}$ both drugs were equally effective in converting AF to sinus rhythm [8].

An alternative for the conversion of recent-onset (duration of 3 to $48 \mathrm{~h}$ ) AF is Vernakalant, a new drug that is still under FDA's investigation for approval. This drug pharmacologically differs from others agents because its action seems to be directed selectively to potassium currents of the atria, resulting in a prolongation of atrial action potential not significantly affecting the QT interval or ventricular refractory period. Therefore, proarrhythmic effects may be minimized. The benefits of early cardioversion include a reduction in the risk of recurrent $\mathrm{AF}$ and less need for long-term anticoagulation or delayed electrical cardioversion. The major benefit of amiodarone on recent-onset $\mathrm{AF}$ is slowing the heart rate within the first 90 minutes, which may provide symptom relief in some patients, including those who did not convert to sinus rhythm.

\section{Conclusion}

Amiodarone may not the best first line durg for every AF patient and, there is little justification for using amiodarone in the ED to convert AF in a timely fashion in WPW patients. Electrical cardiversion should be the first choice in this clinical scenario. A randomized controlled clinical trial directly comparing amiodarone and procainamide in WPW patients is necessary.

\section{References}

1. Bauernfeind RA, Wyndham CR, Swiryn SP, Palileo EV, Strasberg B, Lam W, Westveer D, et al. Paroxysmal atrial fibrillation in the Wolff-Parkinson-White syndrome. Am J Cardiol. 1981;47(3):562-569.

2. Fitzsimmons PJ, McWhirter PD, Peterson DW, Kruyer WB. The natural history of Wolff-Parkinson-White syndrome in 228 military aviators: a long-term follow-up of 22 years. Am Heart J. 2001;142(3):530-536.

3. Wann LS, Curtis AB, January CT, Ellenbogen KA, Lowe JE, Estes NA, 3rd, Page RL, et al. 2011 ACCF/ 
AHA/HRS focused update on the management of patients with atrial fibrillation (Updating the 2006 Guideline): a report of the American College of Cardiology Foundation/American Heart Association Task Force on Practice Guidelines. Heart Rhythm. 2011;8(1):157-176.

4. Tijunelis MA, Herbert ME. Myth: Intravenous amiodarone is safe in patients with atrial fibrillation and WolffParkinson-White syndrome in the emergency department. CJEM. 2005;7(4):262-265.

5. Simonian SM, Lotfipour S, Wall C, Langdorf MI. Challenging the superiority of amiodarone for rate control in Wolff-Parkinson-White and atrial fibrillation. Intern Emerg Med. 2010;5(5):421-426.
6. Perticone F, Cuda G, Spadea F, Pintaudi C, Tropea R. Malignant ventricular arrhythmia in the Wolff-Parkinson-White syndrome during amiodarone treatment. Clin Cardiol. 1987;10(8):477-480.

7. Schutzenberger W, Leisch F, Gmeiner R. Enhanced accessory pathway conduction following intravenous amiodarone in atrial fibrillation. A case report. Int J Cardiol. 1987;16(1):93-95.

8. Chevalier P, Durand-Dubief A, Burri H, Cucherat M, Kirkorian G, Touboul P. Amiodarone versus placebo and class Ic drugs for cardioversion of recent-onset atrial fibrillation: a meta-analysis. J Am Coll Cardiol. 2003;41(2):255-262. 